\title{
PixFRET, an ImageJ Plug-in for FRET Calculation That Can Accommodate Variations in Spectral Bleed-throughs
}

\author{
JÉRÔME N. FEIGE, ${ }^{1}$ DANIEL SAGE, ${ }^{2}$ WALTER WAHLI, ${ }^{1}$ BÉATRICE DESVERGNE ${ }^{1}$ AND LAURENT GELMAN ${ }^{1 *}$ \\ ${ }^{1}$ Center for Integrative Genomics, NCCR Frontiers in Genetics, University of Lausanne, Switzerland \\ ${ }^{2}$ Biomedical Imaging Group, Ecole Polytechnique Fédérale de Lausanne (EPFL), Lausanne, Switzerland
}

\begin{abstract}
KEYWORDS fluorescence resonance energy transfer; sensitized emission; spectral bleedthrough; ImageJ; PPAR; RXR
\end{abstract}

\begin{abstract}
Fluorescence resonance energy transfer (FRET) allows the user to investigate interactions between fluorescent partners. One crucial issue when calculating sensitized emission FRET is the correction for spectral bleed-throughs (SBTs), which requires to calculate the ratios between the intensities in the FRET and in the donor or acceptor settings, when only the donor or acceptor are present. Theoretically, SBT ratios should be constant. However, experimentally, these ratios can vary as a function of fluorophore intensity, and assuming constant values may hinder precise FRET calculation. One possible cause for such a variation is the use of a microscope set-up with different photomultipliers for the donor and FRET channels, a set-up allowing higher speed acquisitions on very dynamic fluorescent molecules in living cells. Herein, we show that the bias introduced by the differential response of the two PMTs can be circumvented by a simple modeling of the SBT ratios as a function of fluorophore intensity. Another important issue when performing FRET is the localization of FRET within the cell or a population of cells. We hence developed a freely available ImageJ plug-in, called PixFRET, that allows a simple and rapid determination of SBT parameters and the display of normalized FRET images. The usefulness of this modeling and of the plug-in are exemplified by the study of FRET in a system where two interacting nuclear receptors labeled with ECFP and EYFP are coexpressed in living cells. Microsc. Res. Tech. 68:5158, 2005. ๑ 2005 Wiley-Liss, Inc.
\end{abstract}

\section{INTRODUCTION}

Fluorescence resonance energy transfer (FRET) is a technique used to investigate interactions between fluorescent partners as it allows one to go beyond the resolution of light microscopy by providing valuable information on distances between tagged molecules. Indeed, since the energy transfer efficiency is inversely proportional to the power 6 of the distance between the donor and the acceptor, interactions are generally detected if the distance between fluorophores does not exceed $5-10 \mathrm{~nm}$, a proximity only allowed by a direct interaction of the two partners (Sekar and Periasamy, 2003). With the introduction of autofluorescent proteins that can be coupled to a protein of interest to produce a fluorescent chimera (Wouters et al., 2001), FRET has gained great importance among cell biologists as it allows them to directly study protein-protein interactions in the physiological context of a living cell.

A plethora of methods exist to evaluate FRET, depending on the protocol used (sensitized emission, acceptor photobleaching, fluorescence lifetime) and the precision that is pursued (see Berney and Danuser (2003) and Jares-Erijman and Jovin (2003) for reviews). Two important parameters in sensitized emission FRET calculation are (i) the evaluation of spectral bleed-throughs (SBTs), i.e., the amount of light detected in the FRET channel that is not due to energy transfer and (ii) the normalization for differences in donor and acceptor expression levels (Gordon et al., 1998; Xia and Liu, 2001). The main causes of SBT are the emission of the donor in the acceptor channel and the excitation of the acceptor using the donor excitation wavelength. As a first approximation, a constant value is often assigned to the donor and acceptor SBT ratios, which then correspond to the average ratios between the intensities in the FRET and in the donor or acceptor channels, when only the donor or acceptor are present. However, we and others have observed that SBT ratios can vary importantly with fluorophore intensity (Chen et al., 2005; Elangovan et al., 2003). To circumvent the problem of generating inaccurate FRET data by using constant SBT ratios, Elangovan et al. (2003) have developed an elegant algorithm termed pFRET that defines classes of fluorophore intensities to which specific SBT values are attributed. We analyzed here the causes of SBT ratio variations on two different confocal microscopes and demonstrate that these are due to the use of several PMTs for the detection in the donor and the FRET channels. We

\footnotetext{
*Correspondence to: L. Gelman, Center for Integrative Genomics, Université de Lausanne, BEP, CH-1015 Lausanne, Switzerland.

E-mail: laurent.gelman@unil.ch

Received 25 February 2005; accepted in revised form 5 July 2005

Contract grant sponsors: UNIL/EPFL Fund, The National Research Project 50, The Swiss National Science Foundation and The Etat de Vaud. DOI 10.1002/jemt.20215

Published online in Wiley InterScience (www.interscience.wiley.com).
} 
thereafter show that a simple modeling to estimate these SBT ratios as a function of intensity greatly improves the accuracy of FRET calculation.

Another important interest for biologists when analyzing FRET in living cells concerns the location of the interaction studied within the cell or the cell population. We report here the development of an ImageJ plug-in, called "PixFRET," which allows one to calculate and display FRET directly on images by performing a pixel-by-pixel analysis of images and assisting in the determination of SBTs.

The usefulness of the modeling of SBT ratios and of the pixFRET plug-in is exemplified using the enhanced cyan and yellow fluorescent proteins (ECFP and EYFP), the FRET pair which is today the most widely used by biologists to study interactions of proteins in living cells, fused to each partner of a well-established heterodimer: the peroxisome proliferator-activated receptor (PPAR) and the retinoid $\mathrm{X}$ receptor (RXR) (Feige et al., 2005).

\section{MATERIALS AND METHODS Plasmid Constructs and Reagents}

cDNAs encoding mouse PPAR $\alpha$ as well as $R X R \alpha$ were subcloned after PCR amplification into the pEYFP-N1 and pECFP-N1 plasmids (BD Biosciences Clontech, Switzerland) as described previously (Feige et al., 2005). The ECFP-DEVD-EYFP construct and GI262570-FITC were kind gifts of Dr. J.M. Tavaré (Rehm et al., 2002) and Dr. Peterson (DeGrazia et al., 2003), respectively.

\section{Cell Culture and Transient Transfection Assays}

Cos-7 cells were maintained in Dulbecco's modified Eagle's medium supplemented with $10 \%$ fetal calf serum (Gibco/Invitrogen, Switzerland). Penicillin and streptomycin (Gibco) were added to the media at 100 units $/ \mathrm{ml}$ and $100 \mu \mathrm{g} / \mathrm{ml}$, respectively.

Transient transfection assays were performed using Lipofectamine 2000 (Invitrogen, Switzerland). Cells were plated in 4-well LabTek chambered coverglasses (Nunc) for microscopy studies.

\section{Confocal Imaging}

Live cells grown on LabTek chambered coverglasses were washed once with phenol red free Optimem medium (Gibco) and observed in the same medium. Observations were performed at $37^{\circ} \mathrm{C}$ on a TCS SP2 AOBS confocal microscope (Leica, Germany) equipped with four photomultipliers (PMTs) and with a wholemicroscope incubator (Life Imaging Service, Switzerland). Additional experiments were performed on an LSM510 Meta confocal microscope, using the Meta array of PMTs (Zeiss, Germany), and on an Olympus IX70 wide-field microscope equipped with a Polychrome II monochromator (Photonics, USA) set at $420 \mathrm{~nm}$, a Zeiss 488027 filter set (Ex BP 410/16 + 489/ 22 - Em BP 456/17 + 535/44) or an Olympus YFP filter set (BP535/30) and an Imago charge-coupled device (CCD) camera (Photonics, USA). Acquisitions were performed with a $63 \mathrm{X} / \mathrm{NA} 1.2$ water or a $63 \mathrm{X} / \mathrm{NA} 1.4$ oil immersion objective. Quantification of images was performed using either the Leica Confocal Software (LCS) version 2.4 or ImageJ version 1.33 .
For FRET experiments, transfections were performed as described above and expression levels of donor and acceptor proteins were adjusted to similar levels by Western blot. Unless otherwise stated, the three different settings used for the analysis of FRET with the CFP/YFP pair were (i) FRET: Ex $405 \mathrm{~nm} / \mathrm{Em}$ 525-545 nm, (ii) CFP: Ex $405 \mathrm{~nm} / \mathrm{Em}$ 465-485 nm, (iii) YFP: Ex $514 \mathrm{~nm} / \mathrm{Em}$ 525-545 nm. Laser power and detector gain were adjusted in the different channels in order to observe equimolar concentrations of CFP and YFP at equal intensities (equimolar concentrations of CFP and YFP were obtained by expressing a reference fusion protein of CFP and YFP spaced by 475 residues). The analysis of FRET with the FITC/Cy3 pair was performed with the following settings: (i) FRET: Ex 488 nm/Em 565-585 nm, (ii) FITC: Ex 488 nm/Em 510-530 nm, (iii) Cy3: Ex 514 nm/Em 565-585 nm. Settings were kept unchanged for analysis of all samples. Unless otherwise stated, donor emission was detected on the photomultiplier 1 (PMT1) and acceptor and FRET emissions were recorded on PMT2. Donor and acceptor SBTs in the FRET setting were determined on cells expressing the donor or the acceptor alone by calculating the intensity $(I)$ ratios in the appropriate settings $\left(I_{\mathrm{FRET}} / I_{\text {Donor }}\right.$ and $I_{\mathrm{FRET}} / I_{\text {Acceptor, }}$, respectively). Linear and exponential fits were performed using Microsoft Excel and FindGraph, respectively. FRET measured in coexpressing cells was then corrected for SBTs and normalized (NFRET) for expression levels according to the following formula (Xia and Liu, 2001):

$$
\mathrm{NFRET}=\frac{I_{\text {FRET }}-I_{\text {Donor }} \times \mathrm{BT}_{\text {Donor }}-I_{\text {Acceptor }} \times \mathrm{BT}_{\text {Acceptor }}}{\sqrt{I_{\text {Donor }} \times I_{\text {Acceptor }}}}
$$

\section{Program for the Plug-in}

The program PixFRET presented in this paper is a plug-in of the public-domain software ImageJ. ImageJ is a general-purpose image-processing program; it is the Java offspring of the NIH Image software. As a result, it can run on any platform with a Java Virtual Machine (Macintosh, Windows, Unix, etc.). The application and its source are available at http://rsb. info.nih.gov/ij/. PixFRET is freely available at http:// www.unil.ch/cig/page16989.html.

\section{RESULTS \\ SBT Ratios Can Vary as a Function of Fluorophore Intensity}

To study FRET in living cells, we chose two nuclear receptors previously shown to form heterodimers in vitro and in vivo, PPAR and RXR that were, respectively, fused to ECFP (donor) and EYFP (acceptor) (Feige et al., 2005).

An important parameter in FRET experiments is the amount of SBT between channels. To estimate SBTs, average fluorescence intensities in cells expressing only the donor (or the acceptor) were quantified, both in the FRET and in the donor (or acceptor) channels. The ratios between the fluorescence intensities in each channel were calculated after background subtraction. In cells expressing PPAR-CFP alone, the donor SBT ratio, defined as the ratio between the amount of light 

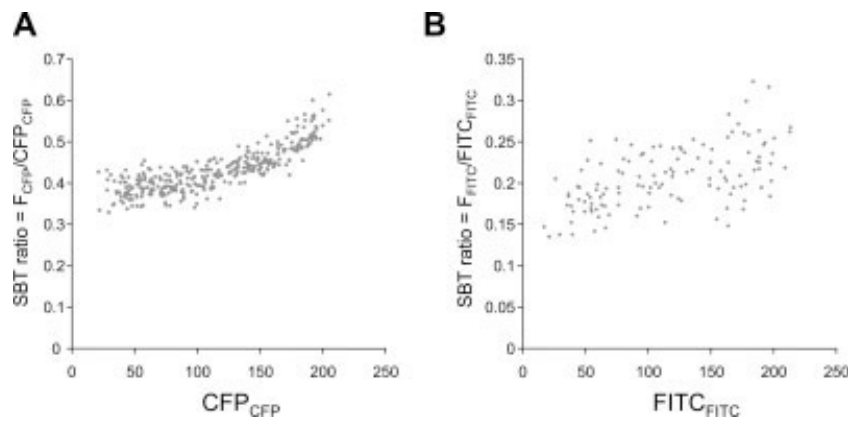

A

B array of PMTs, a very similar increase was observ when the SBT ratio was plotted as a function of fluorophore intensity. However, this donor SBT ratio was constant on a wide-field microscope using a CCD camera (data not shown). It therefore appeared that the dependency of the SBT ratio on fluorophore intensity was restricted to confocal microscopes. To further identify the possible causes leading to this variation, we tested the impact of laser power and PMT gain on SBT ratio variations. Variations of CFP SBT ratios were still observed when the laser was tuned from $15 \%$ to $75 \%$ of its maximum power (data not shown). We then analyzed SBT ratios with four different settings where the gains of the PMTs used for the detection in the donor and FRET channels were set to different voltages. Laser power was adjusted to allow the analysis of the same batch of cells with each setting (Fig. 2A). Interestingly, although the CFP SBT ratio was independent
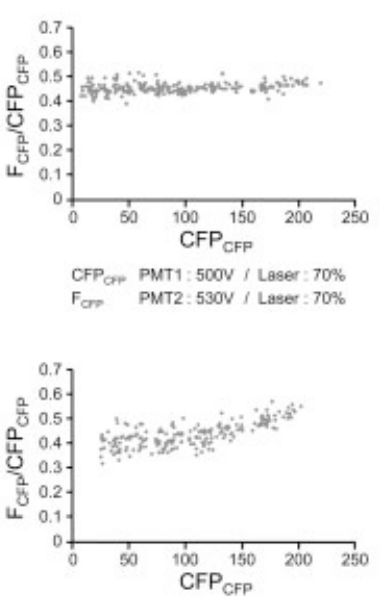

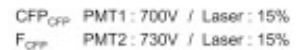
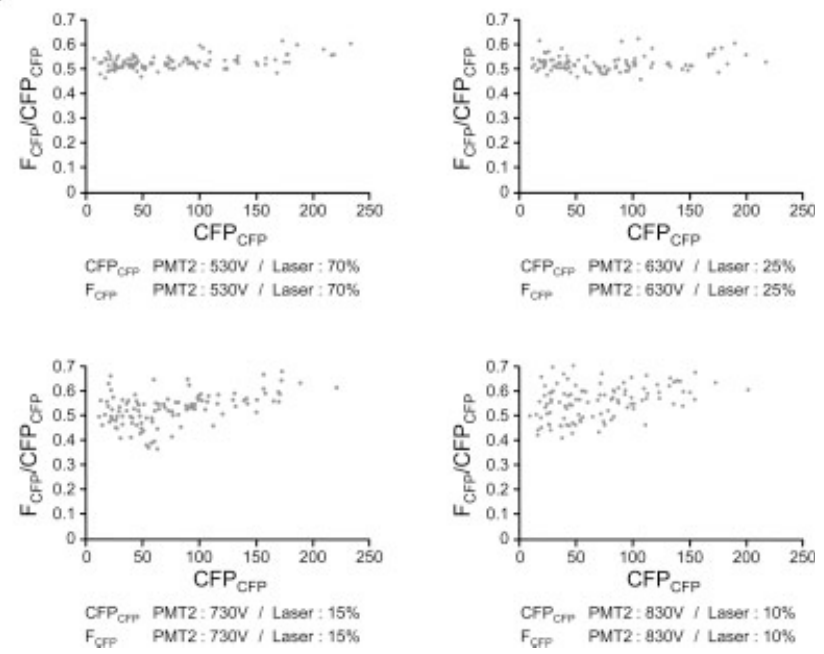

Fig. 2. Influence of PMT gain on the relationship between ECFP SBT ratio and ECFP intensity. Cos-7 cells were transfected with an expression vector for PPAR $\alpha$-ECFP. Fluorescence intensity in the ECFP and FRET settings was measured on at least 100 cells per condition. The percentage of SBT is the ratio between the average FRET and ECFP intensities measured in individual cells. A: The gain and laser power were set as indicated using PMT1 and PMT2 for ECFP (465-485 $\mathrm{nm}$ ) and FRET (525-545 nm) detection, respectively. B: The gain and laser power were set as indicated using PMT2 both for ECFP and FRET detection.
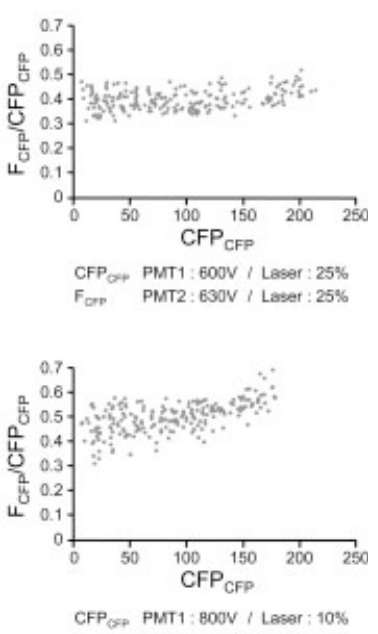

For PMT2 : 830N / Laser : $10 \%$

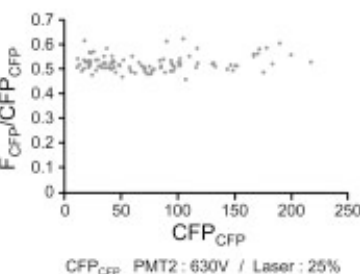

CFP CPP PMT2 : 630N / Laser : 25\%

For PMT2: 630V / Lager: 25\%

of CFP intensity at low PMT gains (500 V donor/530V FRET), this ratio increased with CFP intensity at higher gains. Similar results were obtained when only the donor or the FRET gain were changed, or when both gains were changed and laser power was kept constant (data not shown).

These results suggested that problems in PMT linearity could account for the observed dependency of SBT ratios on fluorophore intensity. While it is more convenient to use two different PMTs for the detection in the donor and FRET channels, we investigated whether the phenomenon could also be observed with one PMT only. When the same PMT was used for both the donor and FRET channels, the SBT ratio was con- 
A
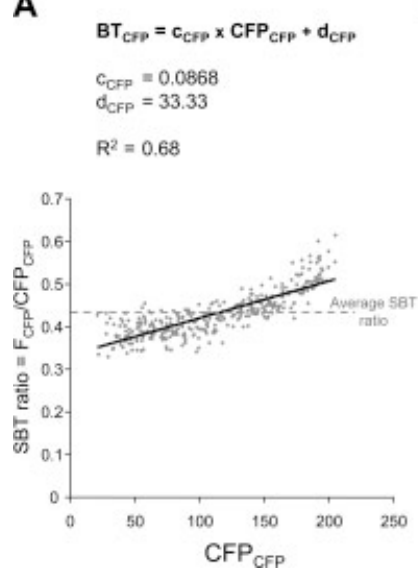

B

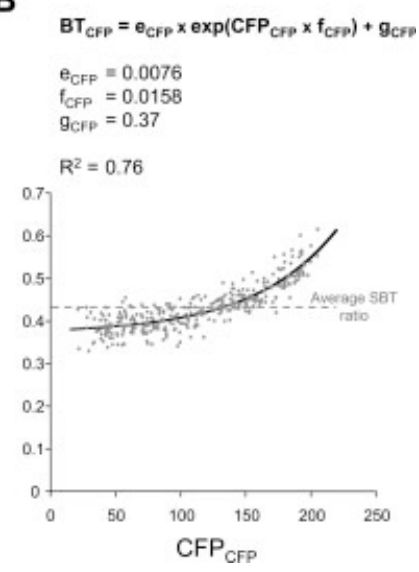

Fig. 3. The ECFP SBT ratio can be fitted as a function of ECFP intensity. The data from Figure 1 were fitted with a linear $(\mathbf{A})$ or an exponential (B) model.

stant, irrespective of the gain applied to the PMT (Fig. 2B). This result suggests that the variations observed with the set-up with two PMTs, or with the array of PMTs, reflect a differential response of the PMTs.

\section{Improving FRET Calculation by Modeling SBT Ratios as a Function of Fluorophore Intensity}

To circumvent the problem of SBT variation, using a unique PMT both for the detection of the donor and of the acceptor seems therefore to be the best solution. However, this imposes to acquire the three channels sequentially because of mechanical movement of the PMT, a time-consuming process that is not compatible with the FRET analysis of rapidly diffusing complexes.

Elangovan et al. (2003) have already reported the variation of SBT ratios as a function of fluorophore intensity and proposed an elegant but only commercially available algorithm to tackle this problem. Alternately, we propose an easily applicable method that consists in modeling SBT ratios as a function of fluorophore intensity. For the problem reported herein, the CFP SBT ratio can be fitted as a function of CFP intensity either with a linear or an exponential model (Fig. 3). These models are then used to estimate the SBT ratio corresponding to each donor intensity and can be implemented in the NFRET formula described by Xia and Liu (2001) as follows: where $F, \mathrm{CFP}$, and YFP are the intensities measured with the FRET, CFP, and YFP settings (correspond to F, D, and A in the nomenclature proposed by Gordon et al. (1998)), $a$ and $b$ are the average donor and acceptor SBT ratios, and $c, d, e, f$, and $g$ are the constants determined by the fitting of the SBT ratio, according to Figure 3.

To validate this approach, the three FRET calculation methods have then been compared with the same set of data (Fig. 4). Cells were transfected with expression vectors for ECFP and EYFP (negative control), ECFP fused to EYFP (positive control), or PPAR-ECFP and RXR-EYFP. The three formulas give similar results for both the positive and negative FRET controls as, under these experimental conditions, variations between cells are small and all fluorescence intensities are close to the average of the population used to calculate SBT ratios (Fig. 4A). The linNFRET values are slightly higher because of the underestimation of the ECFP SBT for both low and high ECFP intensities (see Fig. 3A). The most dramatic effect is seen when NFRET is calculated for the interaction between the two nuclear receptors. Indeed, the more accurate linNFRET and expNFRET calculations significantly reduce standard deviation, with a more pronounced effect for expNFRET. To better characterize the reduction of the variability of the measurements when modeling SBT ratios, we plotted the difference between expNFRET or linNFRET and NFRET as a function of fluorophore intensity (Fig. 4B). In both cases, the highest variation was observed for extreme ECFP values for which using an average SBT ratio leads to a great under- or overestimation of the correction factor (Figs. 4B and 4C). The results obtained with linNFRET and expNFRET are hence not only more precise, but also improve the statistical properties of the data, allowing the user to compare more accurately mild changes. We also tried to fit the donor SBT with a second order polynomial model rather than an exponential growth, but this model greatly overestimates SBT values when donor intensities are very low or very high (data not shown).

\section{Pixel-by-Pixel FRET Analysis and Visualization}

Cells are highly organized and protein distribution as well as interactions are often limited to specific compartments. It is hence crucial for biologists to be able to map interactions with precision within a cell or a population of cells to better understand the spatial organization of cellular activities. We therefore developed an ImageJ plug-in called "PixFRET" that allows one to generate normalized FRET images, by computing pixel

$$
\begin{gathered}
\text { NFRET }=\frac{F_{\mathrm{CFP}+\mathrm{YFP}}-\mathrm{CFP}_{\mathrm{CFP}+\mathrm{YFP}} \times a-\mathrm{YFP}_{\mathrm{CFP}+\mathrm{YFP}} \times b}{\sqrt{\mathrm{CFP}_{\mathrm{CFP}+\mathrm{YFP}} \times \mathrm{YFP}_{\mathrm{CFP}+\mathrm{YFP}}}} \\
\operatorname{linNFRET~}=\frac{F_{\mathrm{CFP}+\mathrm{YFP}}-\mathrm{CFP}_{\mathrm{CFP}+\mathrm{YFP}} \times\left(c_{\mathrm{CFP}} \times \mathrm{CFP}_{\mathrm{CFP}+\mathrm{YFP}}+d_{\mathrm{CFP}}\right)-\mathrm{YFP}_{\mathrm{CFP}+\mathrm{YFP}} \times b}{\sqrt{\mathrm{CFP} P_{\mathrm{CFP}+\mathrm{YFP}} \times \mathrm{YFP}_{\mathrm{CFP}+\mathrm{YFP}}}} \\
\operatorname{expNFRET}=\frac{F_{\mathrm{CFP}+\mathrm{YFP}}-\mathrm{CFP}_{\mathrm{CFP}+\mathrm{YFP}} \times\left(e_{\mathrm{CFP}} \times \exp \left(\mathrm{CFP}_{\mathrm{CFP}+\mathrm{YFP}} \times f_{\mathrm{CFP}}\right)+g_{\mathrm{CFP}}\right)-\mathrm{YFP}_{\mathrm{CFP}+\mathrm{YFP}} \times b}{\sqrt{\mathrm{CFP}}}
\end{gathered}
$$


A

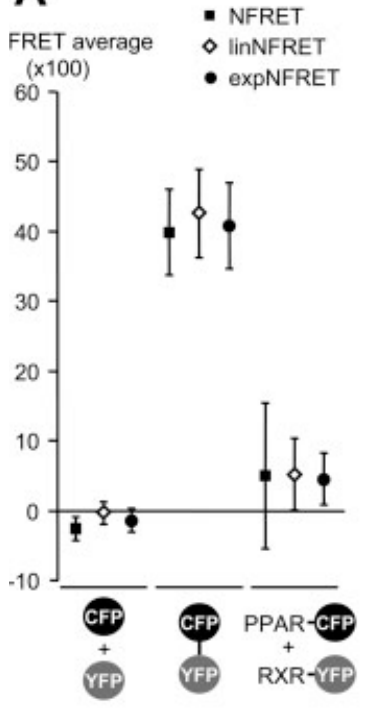

C

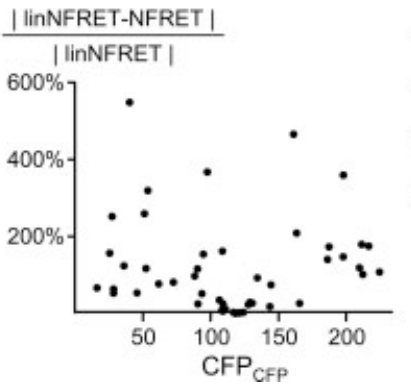

B
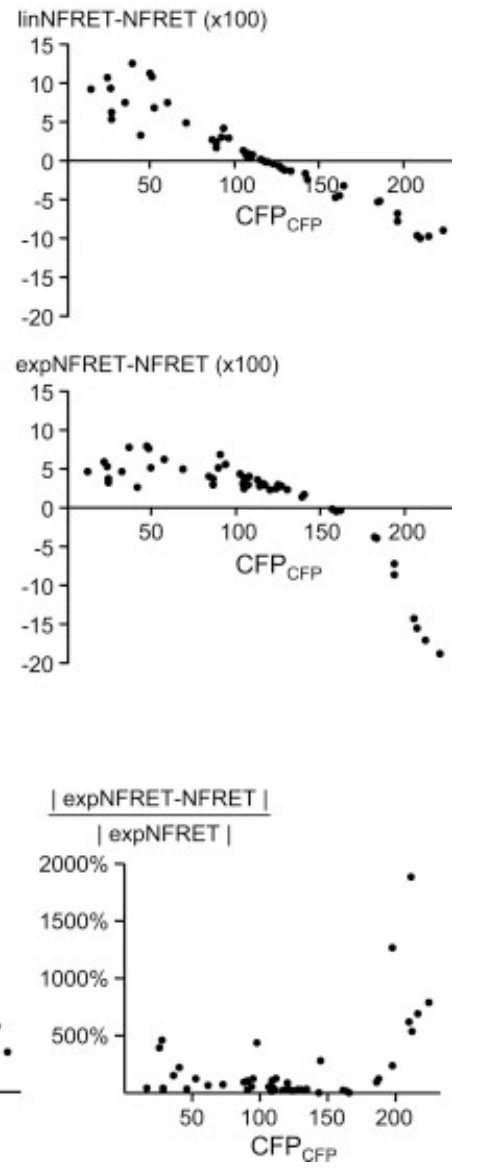

Fig. 4. linNFRET and expNFRET reduce NFRET variability. Cos7 cells were transfected with expression vectors for ECFP and EYFP, ECFP fused to EYFP, or PPAR $\alpha$-ECFP and RXR $\alpha$-EYFP. Fluorescence intensity in the ECFP, EYFP, and FRET settings was measured on at least 50 cells. A: FRET was calculated according to the three formulas described in the text. B: The difference between linNFRET or expNFRET and NFRET calculated for the PPAR/RXR pair was plotted as a function of ECFP intensity. C: The percentage error between NFRET and linNFRET or expNFRET calculated for the PPAR/RXR pair was plotted as a function of ECFP intensity.

by pixel the images of a sample acquired in a threechannel setting. The plug-in allows one to either enter manually SBT parameters for the donor and the acceptor, or determine these values online using stacks of FRET and donor or acceptor images. PixFRET is freely available on our website (http://www.unil.ch/cig/ page16989.html) together with a detailed user's guide.

The PixFRET plug-in requires different stacks of images: (i) for FRET analysis, a stack of three images taken in the FRET, Donor, and Acceptor settings, (ii) for Donor SBT determination, a stack of two images of cells expressing the Donor only and taken in the FRET and Donor settings, (iii) for Acceptor SBT determination, a stack of two images of cells expressing the Acceptor only and taken in the FRET and Acceptor settings.

When launching PixFRET, an interface appears where all parameters for FRET calculation and dis- play can be set (Fig. 5). The "Donor SBT" or "Acceptor SBT" tabs allow one to evaluate SBT parameters (Fig. 5A). The user is first invited to draw a region outside cells to determine the background in each image of the stack. Then, one or several regions of interest on the cells have to be drawn to allow PixFRET to plot SBT ratios as a function of fluorophore intensity. PixFRET then calculates the mean SBT, or the parameters corresponding to the linear or exponential fit of the data. The user can drag a sliding window on the plot to define on which set of points the calculation is to be performed and may then rescale the graph to the selected area by clicking the "Zoom" button. It is also noteworthy that all parameters, including background (BG) values, can be directly entered manually by the user. Next, the stack with cells expressing both the donor and the acceptor should be opened and background should be determined in the FRET tab (Fig. 5B) by drawing a region outside cells. Backgrounds may also be modified manually to adjust the threshold above which NFRET is calculated. Indeed, the software operates NFRET calculation only when (i) the local mean pixel value, which is calculated by averaging the values of the pixel of interest and that of its eight surrounding neighbors, is above the mean background of the image in all three images, and (ii) when the product of the mean pixel values for donor and acceptor is above the product of the backgrounds in the donor and acceptor images. These conditions were introduced to discard points in the background that preclude the visualization of FRET within the cell by generating aberrantly high FRET values because of normalization. All background values may also be changed by a common multiplication factor which can be specified in the "Threshold Correction Factor" field. The program also offers the option to filter the images with a Gaussian blur, the standard deviation of the blur allowing the user to tune the smoothing effect. Blurring notably reduces the noise and improves the resulting image by preventing aberrant FRET values in the background. Finally, the normalization method has to be selected. Indeed, the FRET values for each pixel can be divided by the value in the same pixel of the donor intensity, the acceptor intensity, the product of donor and acceptor intensities, or the square root of the product of donor and acceptor intensities. Clicking on the "Compute FRET" button will generate the normalized FRET images, but also will log all parameters to a text file which will be reloaded when the plug-in is relaunched.

FRET and NFRET are calculated only if pixel values in each image are above a given threshold. Otherwise the value is set to zero in the computed image and the pixel is displayed in blue in order to better visualize all the FRET values of the image (Fig. 6B). Indeed, even if within a cell the average FRET value is positive, some pixels may have negative values, especially when the FRET efficiency is low. Since the output image is displayed with a 256 grayscale lookup table, the background, which corresponds to a value of zero, may appear in gray (and not black as intuitively expected) if negative pixels are present in the image, thereby precluding a good visual examina- 
A

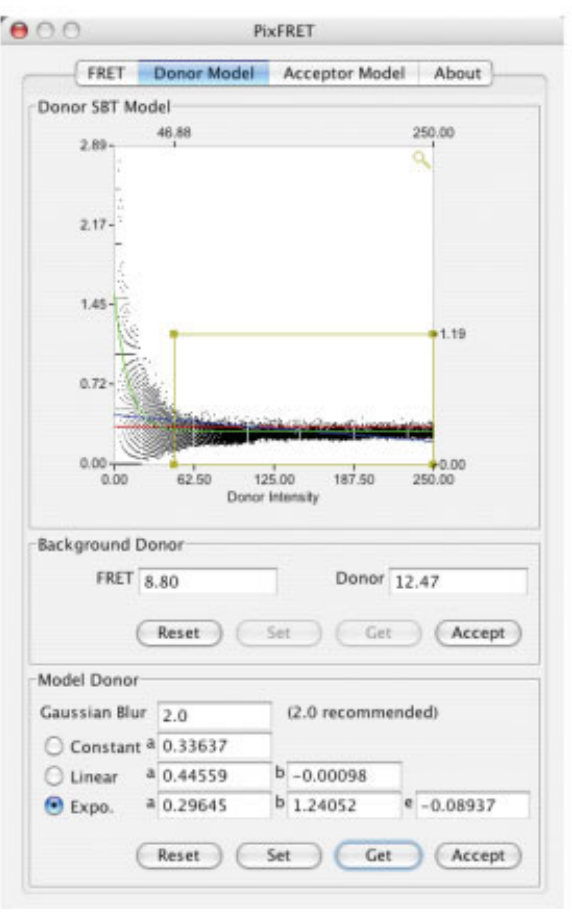

B

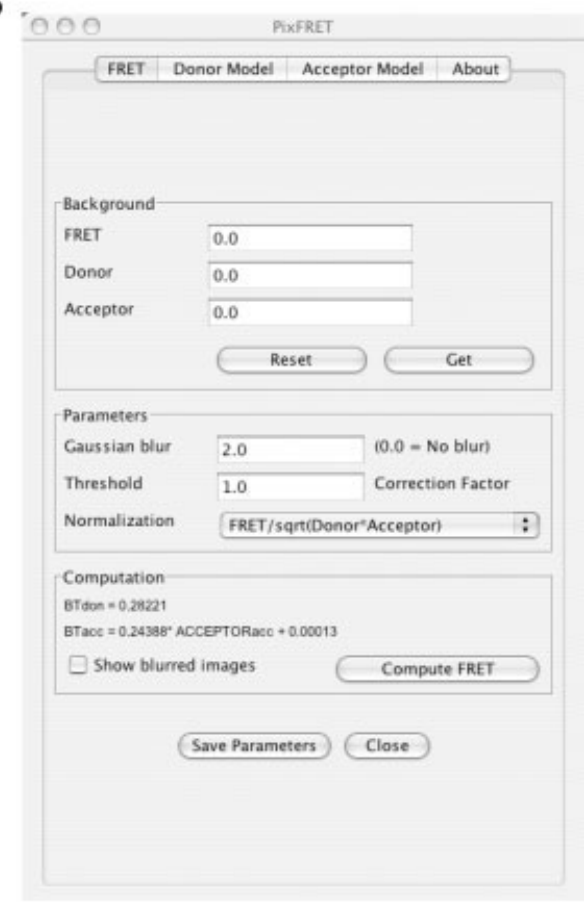

Fig. 5. pixFRET plug-in interfaces. A: SBT determination interface. B: FRET interface. [Color figure can be viewed in the online issue, which is available at www.interscience.wiley.com.]

tion of the generated FRET image. The background pixels are hence automatically colorized in blue using a look-up table designed for this purpose.

\section{Test of the PixFRET Plug-in}

To validate the tools described above, we performed a pixel-by-pixel analysis of FRET by computing an image of two cells expressing different amounts of PPARECFP and RXR-EYFP (Fig. 6) and compared the impact of using constant or fitted SBT ratios on FRET display. Since these cells express the same ECFP and EYFP fusion proteins, both of them should exhibit similar normalized FRET values. When constant SBT ratios are used, FRET efficiency appears higher in cell 2 than cell 1 (Figs. 6B and 6C, left panels), whereas the use of the expNFRET formula generated an image where the two cells display similar intensities (Figs. 6B and $6 \mathrm{C}$, right panels). This visual appreciation of the advantage of using expNFRET was confirmed when the distributions of pixel intensities within each cell were plotted as histograms and average FRET values calculated (Fig. 6D). When constant SBT ratios were used, the average normalized FRET value in cell 1 was 4.9 , versus 13.8 in cell 2 , whereas the use of expNFRET generated closer mean values (9.6 for cell 1 versus 7.4 for cell 2). Indeed, when using an average SBT ratio for normalized FRET calculation (see formula (1)), most donor SBT values are underestimated in cell 2, where CFP levels are high, and overestimated in cell 1 where CFP levels are low. This is why overall and consistent with what was observed at the cell population level in Figure 4, the use of the exponential fit allows a better normalization of the data by reducing the variability of
FRET signals generated from different ranges of fluorophore intensity.

\section{DISCUSSION}

FRET is a technique whose use is rapidly expanding among cell biologists as it provides very valuable information about physical interactions between molecules within cells. Numerous procedures exist and have been used to determine FRET in living cells, each of them having advantages and drawbacks (Berney and Danuser, 2003), and we are still in a phase where the robustness of the methods has to be improved (van Rheenen et al., 2004). One important issue that has received only limited attention so far is the variation of SBT ratios as a function of fluorophore intensity when performing sensitized emission FRET. Using the most widely used fluorophore pair in cell biology, i.e. ECFP and EYFP, we show that under some common technical circumstances, these ratios are not constant and can vary with fluorophore concentration. In cases where the variations of fluorophore concentrations are small, assuming constant SBT ratios may be sufficient to get data of satisfactory accuracy. However, when these SBT ratios vary importantly and thereby clearly bias FRET calculation, and when only small variations in FRET efficiency are expected between cells or samples, solutions to tackle this problem are required.

Variations in SBT ratios as a function of fluorophore intensity could be assigned here to a differential response of the PMTs used for detection in the Donor and FRET channels. Although using a unique PMT both for the detection of the donor and of the 


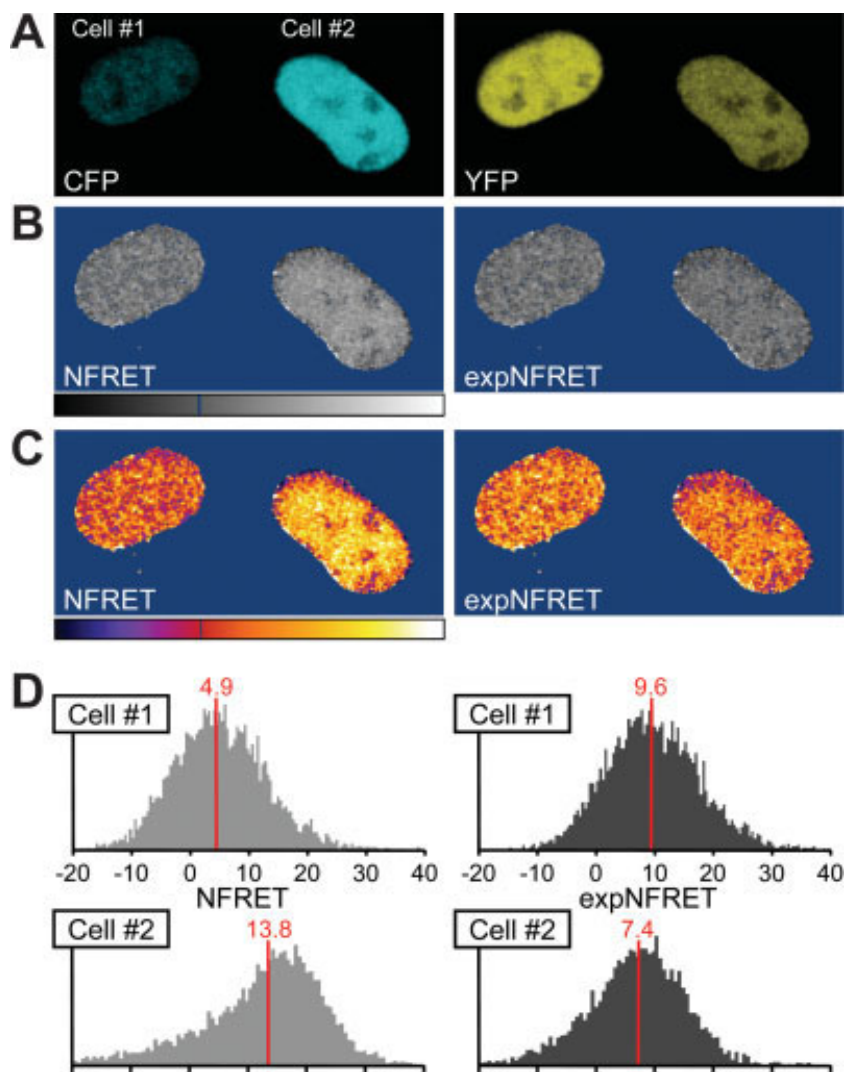

Fig. 6. expNFRET reduces intercellular variability in pixel-bypixel analyses. Cos-7 cells were transfected with expression vectors for PPAR $\alpha$-ECFP and RXR $\alpha$-EYFP. A: Images of two cells expressing both PPAR-ECFP and RXR-EYFP in the CFP and YFP setting. A Gaussian blur of 1 was applied to the original image. B: NFRET and expNFRET images generated by the PixFRET plug-in. C: Same as (B) but the cells are pseudocolorized with a different look-up table to better visualize intensity differences. D: Distribution of pixel intensities within the two cells when the NFRET or expNFRET methods are used. The red bar and the number indicate the mean intensity over the entire cell.

acceptor could circumvent this problem, the required mechanical movement of the PMT (or of the filter cube wheel in other set-ups) implies that the images in the FRET, the donor, and the acceptor channels are acquired sequentially. While this approach is well applicable to fixed samples or to slowly diffusing or immobile proteins, it is more problematic for the study of interactions of highly mobile proteins such as PPARs (Feige et al., 2005). Scanning each channel between lines, which can only be performed with two separate PMTs, therefore, limits the diffusion of the interacting complexes during the time of scanning. It is noteworthy that on a different microscope, using an array of PMTs, the same problem was encountered. Hence, our results are of general interest to scientists performing FRET on a confocal microscope. Interestingly, Chen et al. (in press) have also observed variations in SBT ratios when performing FRET with a 2-photon excitation set-up, and consistent with our study, they did not observe any variation of the SBT ratios on a wide-field microscope, using a CCD camera detection.
In cases where SBT ratios vary as a function of fluorescence intensity, we showed that FRET calculation can be improved by modeling these variations. This is particularly important when fluorophore intensities vary greatly between cells. We demonstrated here that the expNFRET method reduces the variability of the FRET values calculated for the PPAR/RXR interaction. Although in our case, the exponential modeling generated the best results, other fits might be tested in situations where SBT ratios vary differently.

Cells are highly organized and protein distribution as well as interactions are often limited to specific compartments. It is hence of importance to be able to map FRET precisely within cells to better characterize the mode of action of interacting proteins. To achieve this goal, we developed a plug-in called PixFRET for the ImageJ software that generates an image where NFRET is calculated for each pixel. The plug-in allows one to directly determine the SBT parameters from images acquired when only the donor or the acceptor are present. As various normalization methods have been proposed (Gordon et al., 1998; Xia and Liu, 2001), PixFRET allows the user to choose the type of normalization desired. To our knowledge, this is the first freely available program that offers such possibilities and the source files will be available upon request, allowing users to program specific SBT ratio modeling methods according to their needs.

In conclusion, we uncovered the bias that the use of several PMTs may introduce when performing FRET experiments. We show that in cases where a set-up with two PMTs or an array of PMTs is preferred or required, this bias can be circumvented by a simple modeling of SBT ratios. We also developed a user-friendly and free interface, called PixFRET, that allows a simple and rapid determination of SBT parameters and to display normalized FRET images. Altogether, these results and the PixFRET plug-in will help cell biologists to increase the precision of FRET analyses conducted on confocal microscopes as well as the visualization and localization of interactions in living cells.

\section{ACKNOWLEDGMENTS}

We thank Dr. Claude Berney for valuable discussions and for the reading of the manuscript, Dr. Nathalie Garin for access to the ISREC imaging platform, Drs. Peterson and DeGrazia for the gift of the GI-FITC ligand, and Dr. Tavaré for the gift of the EYFP-ECFP construct.

\section{REFERENCES}

Berney C, Danuser G. 2003. FRET or no FRET: a quantitative comparison. Biophys J 84:3992-4010.

Chen Y, Elangovan M, Periasamy A. 2005. FRET data analysis: the algorithm. In: Periasamy A, Day RN, editors. Molecular imaging: FRET microscopy and spectroscopy. Oxford: Oxford University Press (in press).

DeGrazia MJ, Thompson J, Heuvel JP, Peterson BR. 2003. Synthesis of a high-affinity fluorescent PPARgamma ligand for highthroughput fluorescence polarization assays. Bioorg Med Chem 11: 4325-4332.

Elangovan M, Wallrabe H, Chen Y, Day RN, Barroso M, Periasamy A. 2003. Characterization of one- and two-photon excitation fluorescence resonance energy transfer microscopy. Methods 29: $58-73$. 
Feige JN, Gelman L, Tudor C, Engelborghs Y, Wahli W, Desvergne B. 2005. Fluorescence imaging reveals the nuclear behavior of PPAR/RXR heterodimers in the absence and presence of ligand. $J$ Biol Chem 280:17880-17890.

Gordon GW, Berry G, Liang XH, Levine B, Herman B. 1998. Quantitative fluorescence resonance energy transfer measurements using fluorescence microscopy. Biophys J 74:2702-2713.

Jares-Erijman EA, Jovin TM. 2003. FRET imaging. Nat Biotechnol 21:1387-1395.

Rehm M, Dussmann H, Janicke RU, Tavare JM, Kogel D, Prehn JH. 2002. Single-cell fluorescence resonance energy transfer analysis demonstrates that caspase activation during apoptosis is a rapid process. Role of caspase-3. J Biol Chem 277:24506-24514.

Sekar RB, Periasamy A. 2003. Fluorescence resonance energy transfer (FRET) microscopy imaging of live cell protein localizations. J Cell Biol 160:629-633.

van Rheenen J, Langeslag M, Jalink K. 2004. Correcting confocal acquisition to optimize imaging of fluorescence resonance energy transfer by sensitized emission. Biophys J 86:2517-2529.

Wouters FS, Verveer PJ, Bastiaens PI. 2001. Imaging biochemistry inside cells. Trends Cell Biol 11:203-211.

Xia Z, Liu Y. 2001. Reliable and global measurement of fluorescence resonance energy transfer using fluorescence microscopes. Biophys J 81:2395-2402. 